\title{
Editorial
}

\section{Protocolos de actuación clínica en el traumatismo craneoencefálico (TCE) leve. Comentario a la publicación de las guías de la Sociedad Italiana de Neurocirugía}

\author{
J.Sahuquillo
}

Entre un 7 - 10\% de los pacientes atendidos en los servicios de urgencias en países como EEUU y Canadá son pacientes con un traumatismo craneoencefálico (TCE). La mayoría de estos pacientes presentan un TCE leve sin pérdida de conciencia ni alteraciones neurológicas detectables. En otros, existe pérdida de conciencia, desorientación o signos de amnesia postraumática. Aunque en España las cifras disponibles son poco fiables, es muy probable que la frecuencia sea superior a la indicada por dos razones: 1) la incidencia de accidentes de tráfico en nuestro país es superior a la mayoría de países de Europa y EEUU y 2) una mayor tendencia de los afectados a acudir a un hospital después de un TCE leve.

En muchos centros de referencia, el neurocirujano es consultado para valorar a estos pacientes sólo cuando existe pérdida de conciencia, mientras que en otros, es el neurocirujano quien todavía realiza el cribaje de estos pacientes. El número estimado de pacientes con un TCE leve atendidos por un neurocirujano en hospitales públicos españoles en el año 2000 fue superior a 92.000². En los hospitales comarcales, la mayoría de los cuales disponen ya de $\mathrm{TC}$, el problema que plantea la atención a un paciente con un TCE leve es de otra índole. En estos centros, el médico que atiende a estos pacientes suele ser un cirujano general, un traumatólogo, un anestesiólogo, un neurólogo, un médico de emergencias o un especialista en medicina interna. El problema fundamental en este nivel asistencial radica en decidir a qué pacientes y en qué momento debe practicarse una TC cerebral, quién debe evaluar esta exploración y en qué casos el paciente debe ser trasladado a un centro que disponga de servicio de neurocirugía. El riesgo de que una lesión potencialmente tributaria de tratamiento quirúrgico pase desapercibida, aunque reducido, no es despreciable por sus repercusiones sobre la supervivencia y la calidad de vida del paciente afectado. Si a esto se añade la potencialidad de litigio por mala praxis o negligencia ante un acontecimiento de estas características, el escenario para el debate queda claramente definido.

Recibido; 2-01-05. Aceptado:15-01-05
El volumen considerable de pacientes con un TCE leve atendidos en hospitales y el margen de error, no cubierto de forma absoluta por ningún protocolo, ha establecido en muchos centros la práctica de una medicina defensiva, con estrategias más orientadas a evitar la demanda legal que fundamentadas en evidencia científica. Con gran frecuencia el médico que atiende a estos pacientes indica exploraciones neurorradiológicas innecesarias, que sobrecargan los servicios de urgencias y distorsionan su actividad. Los recursos que estos pacientes consumen cuando se aplican políticas excesivamente permisivas con la indicación de TC o de traslado a un centro neuroquirúrgico son considerables y, a menudo, injustificables desde un punto de vista coste-beneficio y de coste-efectividad.

En muchos países se han diseñado, evaluado y seguido diferentes protocolos para el cribaje y manejo de estos pacientes. Estos protocolos, consensuados mayoritariamente con distintas sociedades científicas, en general son refrendados por asociaciones médicas, hospitales y sistemas de salud. Su finalidad principal es, por un lado, detectar precozmente a aquellos pacientes que pueden deteriorar neurológicamente, aunque también pretenden dar cobertura jurídica al médico ante potenciales demandas legales. De los protocolos publicados en los últimos diez años para afrontar este problema, uno de los primeros fueron las denominadas "Guías Italianas" que en este número se presentan traducidas.

Estas guías publicadas originalmente en 1996 en el Journal of Neurological Sciences ${ }^{1}$ fueron seleccionadas, evaluadas y traducidas por el Grupo Español de Neurotraumatología (GEN) a finales del año 2000. El GEN fue un grupo multidisciplinario formado por anestesiólogos, neurocirujanos, médicos de emergencias y especialistas en cuidados intensivos creado en el año 1998 a instancias del Pan-European Advisory Board, organismo cuya misión fue la de elaborar, diseminar y controlar el cumplimiento de guías de práctica clínica en el TCE en el ámbito de la Unión Europea. De los catorce miembros del GEN, cuatro eran neurocirujanos pertenecientes a la Sociedad Española de Neurocirugía (SENEC). El GEN definió como su primer objetivo negociar con la Brain 
Trauma Foundation el permiso de traducción de las guías de manejo del TCE grave publicadas en 1995, obtener financiación para sufragar los costes de los derechos de autor y gestionar su publicación gratuita en España. Todo este proceso fue financiado por la compañía farmacéutica Bayer. Las guías del TCE grave fueron traducidas por los miembros del GEN y se presentaron oficialmente en el congreso de la SENEC de Bilbao en mayo del 2000. Finalizada esta etapa, el GEN definió como un segundo objetivo la preparación de unas recomendaciones consensuadas para el manejo del TCE leve. Posteriormente, y por falta de financiación, este grupo ceso en sus actividades en el año 2001, aunque nunca ha sido disuelto oficialmente.

En el año 1999 el GEN revisó todos los protocolos y recomendaciones existentes para la evaluación y manejo del TCE leve y consideró que el más apropiado para su difusión en nuestro país era el de la Sociedad Italiana de Neurocirugía, conocido como "Guías Italianas". Este protocolo se presenta traducido en este número de Neurocirugía. El GEN solicitó el permiso para traducir y publicar estas guías a la Sociedad Italiana de Neurocirugía. Por varias razones, su publicación se ha retrasado casi dos años más de lo previsto. Este aparente inconveniente, tiene como ventaja el hecho de aportar una cierta perspectiva y permitir hacer valoraciones más ponderadas de la aplicación de estas guías. Una vez decidida su publicación en Neurocirugía, se solicitó a diferentes miembros de las sociedades nacionales de Anestesiología y Reanimación, Emergencias, Cuidados Intensivos, Neurocirugía y a médicos de hospitales comarcales que aportaran sus comentarios y críticas. Los comentarios que se publican en este número, junto a los de los Dres. Servadei y Brambilla de la Sociedad Italiana de Neurocirugía, son los que recibió quien firma este editorial.

Las guías Italianas han sido adoptadas por diferentes sociedades científicas de neurocirugía como la Italiana, la Argentina y la Francesa y han sido recomendadas por la World Federation of Neurosurgical Societies. Por otra parte dos estudios han demostrado su elevada sensibili$\mathrm{dad}^{4,13}$. Estos estudios y las recomendaciones de las distintas sociedades mencionadas refuerzan su aplicabilidad en nuestro medio.

Durante estos últimos años se han diseñado y evaluado también otros protocolos. Entre ellos destacan las guías Escandinavas $^{6}$, las Canadienses y las de New Orleans en EEUU. Las dos últimas son las más aplicadas en el continente Americano. En Europa continental las guías desarrolladas por la Sociedad Italiana de Neurocirugía, con pocas o ninguna modificación, son las mas utilizadas. En Gran Bretaña, sin embargo, las guías del Scottish Intercollegiate Guidelines Network (SIGN) tienen una mayor difusión ${ }^{10}$.
Las guías Italianas dividen a los pacientes con un TCE leve (puntuaciones en la escala de Glasgow de 14 o 15) en tres grupos (Grupo 0,1 y 2) a los que se añade un cuarto grupo $(0-1 \mathrm{R})$ dependiendo de la existencia o ausencia de factores de riesgo (coagulopatía, epilepsia, etc.). Los algoritmos son claros, sencillos de aplicar y fáciles de implementar en centros hospitalarios de cualquier nivel. En un estudio basado en 4536 pacientes con un TCE leve se demuestra que en los pacientes del "grupo 0 ", ninguno presentaba lesiones clínicamente significativas en la TC cerebral ${ }^{13}$. En otro estudio reciente Ibáñez y col. confirman que, con una política más flexible como la seguida en el Hospital Universitario Vall d'Hebron de Barcelona, en la que todos los pacientes del "grupo 0 " fueron explorados por TC cerebral, aunque pueden detectarse lesiones clínicamente no significativas, en ningún caso se detectó una lesión tributaria de tratamiento quirúrgico ${ }^{4}$. Hay que considerar que aunque esta estrategia permisiva puede ser considerada razonable y con un índice coste-efectividad inferior a la observación o al ingreso hospitalario ${ }^{5}$, sólo es un protocolo aceptable para hospitales que dispongan de TC las 24 horas y con un servicio de neurocirugía con guardias de presencia física, de un radiólogo de guardia o de sistemas telerradiológicos ${ }^{8}$. Este escenario sólo es posible en un número muy reducido de hospitales españoles.

Entre los estudios más recientes que abordan este problema hay que destacar la validación externa efectuada por Stille y col. del resultado de aplicar las guías Canadienses y las Americanas a una misma población de pacientes adultos, con puntuaciones en la ECG entre 13-15 y pérdida de conciencia o amnesia postraumática atendidos en hospitales terciarios ${ }^{11,12}$. Aunque este estudio presenta limitaciones que hacen que sus conclusiones no sean fácilmente extrapolables, sí confirman que ambos protocolos son altamente sensibles para detectar a los pacientes con lesiones clínicamente relevantes.

Una de las conclusiones de todas las guías implementadas es que los pacientes con puntuaciones de 13 en la ECG presentan un mayor potencial de deterioro neurológico y de lesiones clínicamente relevantes en la TC cerebral. Por lo tanto estos pacientes deben excluirse de la categoría de TCE leve $\mathrm{e}^{3}$ y a todos se les debe practicar una TC cerebral. En el otro extremo (pacientes con puntuaciones de 14 o 15 en la ECG), existe un grupo reducido pero importante de pacientes que pueden presentar deterioro del nivel de conciencia y/o lesiones cerebrales clínicamente relevantes que pueden, en algunos casos, requerir intervención quirúrgica. Para este grupo numeroso de pacientes, las guías Italianas representan un excelente protocolo para la detección precoz de lesiones evacuables quirúrgicamente.

Para cualquier especialidad involucrada en la asis- 
tencia al TCE resulta evidente que los protocolos de actuación consensuados y con enfoque multidisciplinario son una necesidad para el desarrollo de una práctica asistencial adecuada y para proteger al médico de potenciales demandas. Los neurocirujanos debemos colaborar con todas las especialidades implicadas en la asistencia del paciente traumático y a su transporte a los hospitales de referencia. Las peculiaridades de nuestra red sanitaria nos obliga a discutir su manejo terapéutico con otros especialistas y a consensuar pautas de tratamiento. La publicación de las guías Italianas y de los comentarios de distintas especialidades son un buen punto de partida para discutir, mejorar y evaluar los actuales protocolos asistenciales existentes en nuestro país. Estas guías son un buen protocolo de actuación que permite racionalizar la atención al paciente con un TCE leve, disminuyendo la sobrecarga asistencial y el consumo innecesario de recursos sanitarios.

Como reflexión final, hay que remarcar el comentario que hacen las guías Italianas sobre que "Cualquier protocolo sobre TCE leve debe tener en cuenta los recursos disponibles, no únicamente en los pocos hospitales que disponen de unidades neuroquirúrgicas sino, más importante aún, de todos los hospitales en donde este tipo de pacientes serán tratados"1. Cualquier protocolo que deba aplicarse en nuestro sistema sanitario no debe estar diseñado para un contexto de hospital terciario, sino para toda la población. La mayoría de pacientes con un TCE ocurren en lugares alejados de centros neuroquirúrgicos. Por este motivo las guías deben contemplar y adaptarse a estas circunstancias. Las indicaciones de TC cerebral en cualquier guía deberían ser las mismas para un centro comarcal y para un hospital de referencia. Si bien es cierto que desde el punto de vista coste-beneficio la práctica de una $\mathrm{TC}$ a todo paciente que acude a urgencias puede ser lo más eficaz para optimizar los recursos asistenciales, esta política sólo es aplicable en muy pocos hospitales. Por ello los protocolos de actuación deben llegar a soluciones de compromiso, que permitan aplicar los mismos criterios de actuación al mismo tipo de paciente, independientemente de donde sea atendido. Si esto no se hiciera así, seria poco justificable que un paciente atendido en un hospital comarcal sin TC, no fuera trasladado a un servicio que dispusiera de estos medios. Esto llevaría a pervertir el espíritu de este tipo de recomendaciones y conduciría a practicar una medicina más dirigida a defender al médico de una potencial demanda legal que a optimizar la asistencia de estos pacientes y a reducir el consumo de recursos que generan para el sistema de salud.

Es probable que en un futuro cercano dispongamos de biomarcadores de lesión cerebral que puedan aplicarse de forma rápida en urgencias para el despistaje de lesiones cerebrales relevantes. La proteína S-1008, ha sido ya propuesta por autores escandinavos ${ }^{5,9}$ y podría ser en un futuro una opción si se confirma su elevada sensibilidad y especificidad para detectar lesiones mínimas en pacientes con un TCE leve. Sin embargo, este tipo de estrategias requieren todavía su validación en estudios multicéntricos con un número elevado de casos y su comparación con el patrón oro, la TC cerebral.

Las repercusiones para un sistema sanitario como el español de una estrategia defensiva y excesivamente permisiva con la práctica de exploraciones neurorradiológicas debe ser motivo de reflexión. La publicación de estas recomendaciones en Neurocirugía son un buen principio para estimular el debate que permita consensuar protocolos dirigidos a detectar de la forma más efectiva posible aquellos pacientes con riesgo de presentar lesiones cerebrales importantes con un coste aceptable y sin sobrecargar más a los servicios de urgencias, crónicamente saturados.

\section{Bibliografía}

1. Ambrosio, A., Acampora, S., Arienta, C.,.et al: Guidelines for minor head injured patients' management in adult age. J.Neurol. Sci. 1996; 40: 11-15.

2. Brell, M., Ibañez, J.: Manejo del traumatismo craneoencefálico leve en España: estudio multicéntrico nacional. Neurocirugia 2001; 12: 105-124.

3. Gomez, P.A., Lobato, R.D., Ortega, J.M., De la Cruz, J.: Mild head injury: differences in prognosis among patients with a Glasgow Coma Scale score of 13 to 15 and analysis of factors associated with abnormal CT findings. Brit. 3. Neurosurg. 1996; 10: 453-460.

4. Ibañez, J., Arikan, F., Pedraza, S.,.et al: Reliability of clinical guidelines in the detection of patients at risk following mild head injury: results of a prospective study. J Neurosurg. 2004; 100: 825-834.

5. Ingebrigtsen, T., Romner, B.: Routine early CT-scan is cost saving after minor head injury. Acta Neurol.Scand. 1996; 93: 207-210.

6. Ingebrigtsen, T., Romner, B., KockJensen, C.: Scandinavian guidelines for initial management of minimal, mild, and moderate head injuries. J. Trauma 2000; 48: 760766.

7. Lapierre, F.: Guidelines concerning severe cranial trauma. French Society of Neurosurgery. Neurochirurgie 1998; 44: 132-135.

8. Poca, M.A., Sahuquillo, J., Domenech, P.,.et al: Use of teleradiology in the evaluation and management of headinjured patients. Results of a pilot study of a link between a district general hospital and a neurosurgical referral center. Neurocirugia 2004; 15: 17-35.

9. Romner, B., Ingebrigtsen, T.: High serum S-100B levels for trauma patients without head injuries. Neurosurgery $2001 ; 49: 1490$. 
10. Scottish Intercollegiate Guidelines Network (SIGN): Early management of patients witha head injury, 2000.

11. Smits, M., Dippel, D.W., de Haan, G.G.,.et al: External validation of the Canadian CT Head Rule and the New Orleans Criteria for CT scanning in patients with minor head injury. JAMA 2005; 294: 1519-1525.

12. Stiell, I.G., Clement, C.M., Rowe, B.H.,.et al: Comparison of the Canadian CT Head Rule and the New Orleans Criteria in patients with minor head injury. JAMA 2005; 294 : 1511-1518.
13. Viola, L., Zotta, D., Martino, V., Barbato, R., Schisano, G.: Minor head injuries: One year experience according to the new Italian guideline. Acta Neurochir. 2000; 142: 12811285 .

\section{J.Sahuquillo}

Coordinador del Grupo Español de Neurotraumatología (GEN)

Correspondencia postal: Juan Sahuquillo. Servicio de Neurocirugía. Hospital Universitario Vall d'Hebron. Paseo del Vall d'Hebron 119-129. 08035 Barcelona 